\title{
Living anionic polymerization of a block copolymer and the preparation of superhydrophobic surfaces based on the method of phase separation
}

\author{
Polymer Journal (2013) 45, 125-128; doi:10.1038/pj.2012.137; published online 4 July 2012
}

The wettability of solid surfaces is a subject of intense research today because of its potential applications in daily life and in industry, ${ }^{1-3}$ such as its use in corrosion resistance, stainresistant textiles, drag-reduction, inhibition of snow or ice adhesion, biomedical applications and anti-bacterial paints. The surfaces with water contact angles (WCAs) $>150^{\circ}$ and rolloff angles $<10^{\circ}$ are called superhydrophobic surfaces. $^{4-7}$ Many natural organisms exhibit inherent superhydrophobicity, for example, lotus leaves, rose petals, water strider legs and the skins of some types of fish. ${ }^{8,9} \mathrm{~A}$ large number of artificial superhydrophobic surfaces have been prepared with our in-depth understanding of the chemical compositions and the geometric structures of these biological surfaces. For water repellency, surface roughness and low surface energy are the two essential factors. ${ }^{10-13}$ Generally, superhydrophobic surfaces are made using the two approaches: the application of low surface-energy coatings to modify the roughness of surfaces and the fabrication of surfaces with micro-/nano-rough structures using low surface-energy materials. ${ }^{14-16}$

Living anionic polymerization is an effective route to fabricate polymers with welldefined molecular architectures compared with other polymerization methods. Owing to the incorporation of functional groups into the polymer chains, these polymers always exhibit high-performance characteristics, ${ }^{17}$ such as hydrophilicity, hydrophobicity, biocompatibility and adhesion. One application of living anionic polymerization is to form block copolymers. Under the appropriate conditions, block copolymers are known to generate ordered structures with nanometer-scale periodicity through microphase-separation. ${ }^{18}$ Moreover, using selective solvent mixtures could induce these copolymers to phase separate. ${ }^{19}$ Generally speaking, the phase separation method is a simple and low-cost approach to fabricate superhydrophobic surfaces.

In this work, polystyrene-block-polymethyl methacrylate (PS-b-PMMA) block copolymers were synthesized by sequential living anionic polymerization with $n$-butyl lithium ( $n$-BuLi) as the initiator, and the superhydrophobic surfaces were obtained by casting the PS-b-PMMA diblock copolymer from the mixed solvents of ethanol and tetrahydrofuran (THF). Fractal-like hierarchical micro- or nano-rough structures were observed on the copolymer surfaces using scanning electron microscopy. The diameters of the spherical particles and the roughness of the copolymer surfaces were controlled by the volume ratio of ethanol. The WCA and contact angle hysteresis of the as-prepared surface were measured to be $156.7^{\circ}$ and $7.1^{\circ}$, respectively, which indicated that the copolymer surfaces had excellent superhydrophobicity and selfcleaning ability. Owing to its flexibility and simplicity, the copolymer may be suitable for fabricating superhydrophobic surfaces for large-scale and practical application.

\section{EXPERIMENTAL PROCEDURE}

The PS-b-PMMA block copolymer was synthesized by sequential living anionic polymerization in cyclohexane at $\sim 5^{\circ} \mathrm{C}$ with $n$ $\mathrm{BuLi}$ as the initiator. Approximately $16 \mathrm{ml}$ of cyclohexane and $n$-BuLi solution $(2.3 \mathrm{M}$ in hexane, $0.24 \mathrm{ml}, 0.552 \mathrm{mmol}$ ) was added into a double-necked reactor flask. After a homogeneous mixture of $n$-BuLi solution and cyclohexane was obtained, $2 \mathrm{ml}$ of styrene $(17.379 \mathrm{mmol})$ was introduced into the reactor. The colorless solvent turned red within seconds. After $60 \mathrm{~min}$, the second monomer, methyl methacrylate $(6.00 \mathrm{ml}, 56.332 \mathrm{mmol})$, was added, the mixture turned light blue within seconds and the mixture was stirred for $75 \mathrm{~min}$. The polymerization was terminated by the addition of a $5 \%(\mathrm{v} / \mathrm{v})$ methanol/water solution. The diblock copolymer was precipitated by pouring the solution slowly into methanol and dried under vacuum.

The copolymer $(0.40 \mathrm{~g})$ was dissolved in mixed solvents $(20 \mathrm{ml})$ of ethanol and THF with various volume ratios from 0 to $6: 4$ at the refluxed temperature. After stirring the copolymer solution for $30 \mathrm{~min}$, a few drops of the copolymer solution were then dropped onto the cleaned glass substrate. Various roughness copolymer films were obtained after drying for $12 \mathrm{~h}$ at ambient temperature.

\section{RESULTS AND DISCUSSION}

Figure 1a shows the relationship between the contents of ethanol and the WCAs. The WCA increased with increasing ethanol content, and it reached a peak of $156.7^{\circ}$ at a content of ethanol of $\sim 60 \%(\mathrm{v} / \mathrm{v})$. Note that it was difficult to deposit a water drop onto the copolymer surface because of the extremely low adhesion of the copolymer surface to water. We observed that water droplets rolled off the copolymer surface, taking dirt (carbon soot) along with them (Figure 1c). These phenomena demonstrated that the copolymer surface exhibited good self-cleaning ability. When the ethanol content was $>60 \%(\mathrm{v} / \mathrm{v})$, most of the copolymer could not dissolve in the mixed solvents. A $5-\mu \mathrm{l}$ distilled-water drop profile on the as-prepared film (ethanol content, $60 \%(\mathrm{v} / \mathrm{v})$ ) is also shown on the left-hand side of Figure $1 \mathrm{~b}$. The right-hand side of Figure $1 \mathrm{~b}$ shows that a copolymer surface without any residual water drop was exhibited when the water droplet was sucked back into the needle, that is, the 


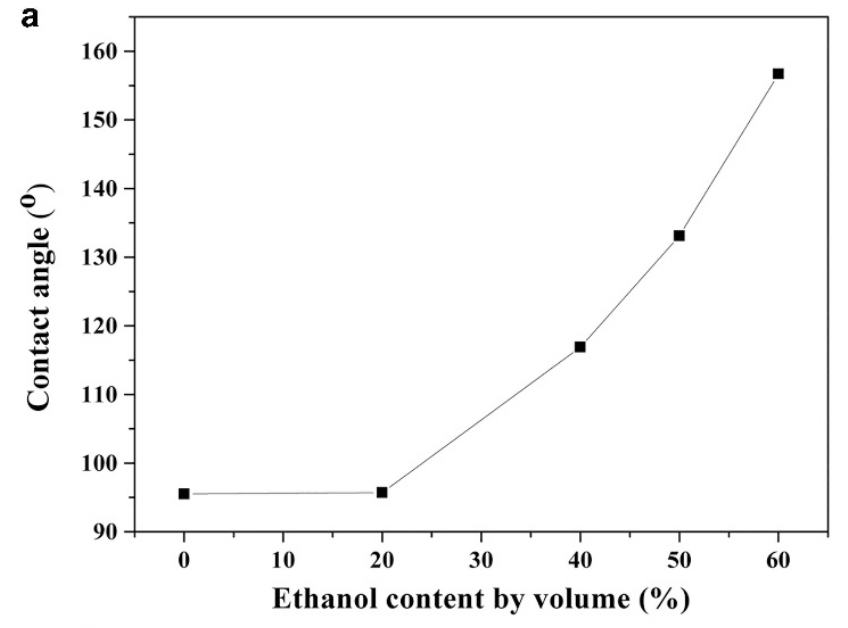

b

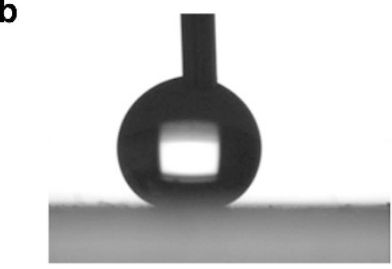

C
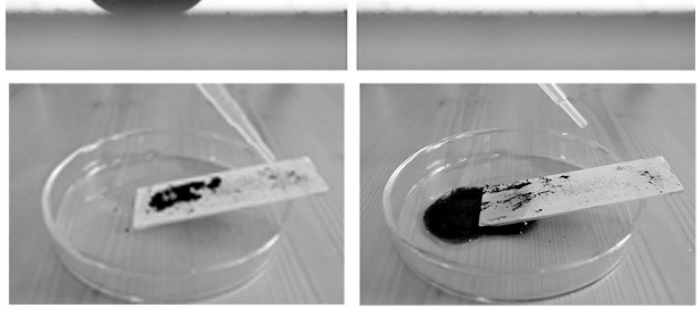

Figure 1 (a) Relationship between the content of ethanol and the water contact angles. (b) A 5- $\mu$ l distilledwater drop profile on the as-prepared film (left); the image of the copolymer surface when the water drop sucked back into the needle (right) (ethanol content, 60\% (v/v)). (c) Image of the copolymer surface (ethanol content, $60 \%(\mathrm{v} / \mathrm{v})$ ) coated with carbon soot (left); image of water droplets rolling off while taking carbon soot along with them (right). A full color version of this figure is available at Polymer Journal online. adhesive force between the water droplet and the superhydrophobic surface was very small. In addition, the contact angle hysteresis of the copolymer surface was $7.1^{\circ}$.

Figure 2 shows the scanning electron microscopy images of the copolymer films obtained from different ethanol content by volume in copolymer solution: (a) $0 \%$; (b) $20 \%$; (c) $40 \%$; (d, e) $50 \%$; (f) $60 \%$. Herein, (e) represents the lower left corner of (d). As shown in Figure 2a, the copolymer surface was nearly flat except for the presence of little pollution. Small protrusions were distributed uniformly on the copolymer surface, and nearly all of the protrusions were separated and not connected to another protrusion. The protrusions were only formed by a monolayer structure on the copolymer surface, and the surface roughness was poor. A large number of white spheres were observed, as shown in Figures $2 \mathrm{~b}-\mathrm{f}$, and many of the white spheres were connected to each other. The white spheres constituted the multilayer rough structures of the copolymer surfaces. However, the particle sizes of the spheres, shown in Figure 2c and in Figure 2e, were different from those shown in Figure $2 \mathrm{f}$, and the particle size exhibited a gradual decrease with an increase in the ethanol content by volume. When the ethanol content was $50 \%$ $(\mathrm{v} / \mathrm{v})$, the particles were aggregated to form layered structures and the overall surface roughness of each layer was not significant (Figure 2d and e). The conical shapes shown in Figure $2 \mathrm{f}$ formed from a small number of
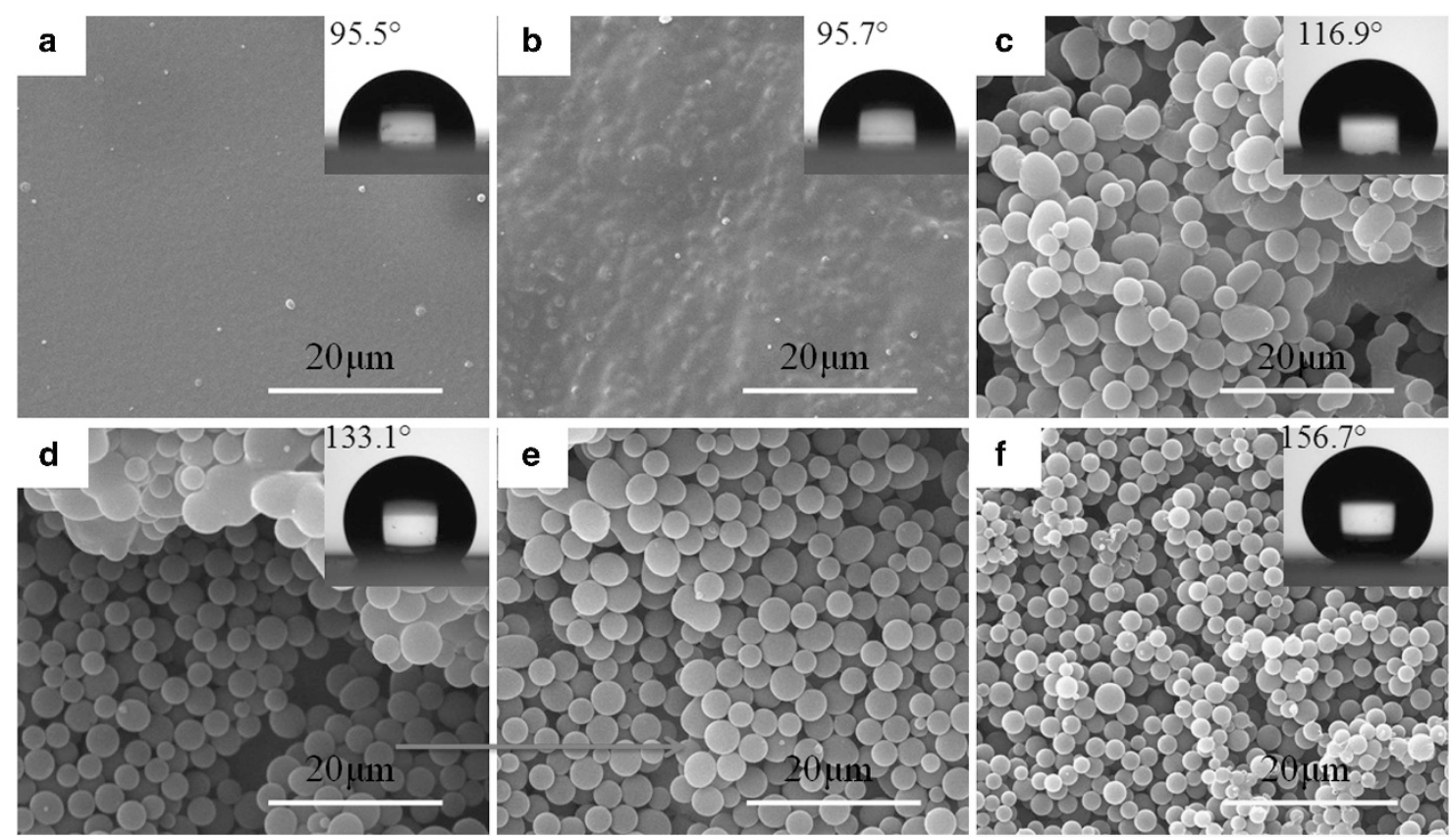

Figure 2 Scanning electron microscopy images of the copolymer films obtained from different ethanol content by volume in a copolymer solution: (a) 0\%; (b) $20 \%$; (c) $40 \%$; (d, e) $50 \%$; (f) $60 \%$. Herein, (e) represents the lower left corner of (d). The inset is the WCA photograph of the copolymer surface. A full color version of this figure is available at Polymer Journal online. 
very bright white particles, and they likely minimized the solid-liquid contact area. ${ }^{11}$ In addition, these shapes could effectively trap air and vigorously support the enwrapped liquid-air interface. A superhydrophobic copolymer surface was created by fractal-like hierarchical micro- to nano-rough structures and the inherent hydrophobicity of PS (the two essential factors to fabricate a superhydrophobic film).

In this study, when evaluating the solubility of the copolymer, THF was observed to be a good solvent compared with ethanol. The solubilities of PS and PMMA in THF and ethanol were measured separately at room temperature. The relative solubilities
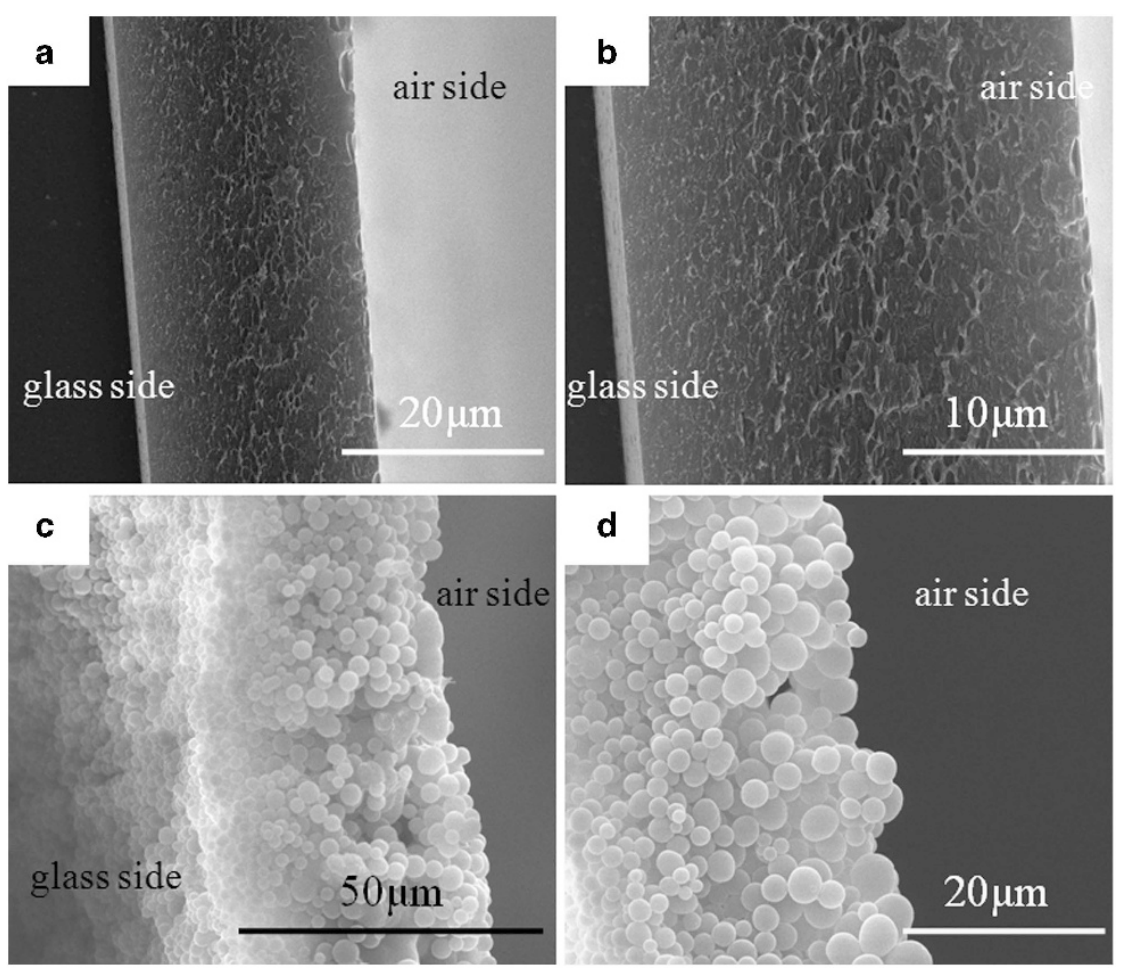

Figure 3 Cross-sectional scanning electron microscopy images of the copolymer films obtained from different ethanol content by volume in a copolymer solution: (a) $0 \%$; (c) $60 \%$; (b, d) were the higher magnification images of $\mathbf{a}$ and $\mathbf{c}$, respectively. of the homopolymers were measured under the same conditions and they were calculated to provide an initial guide. The solubility of PS and PMMA in THF was 253.9 and 14.04 times that in ethanol, respectively. The solubility of PS was 16 and 0.89 times that of PMMA in THF and ethanol, respectively. Moreover, the evaporation rate of THF was 2.7 times that of ethanol at room temperature. Ignoring the influence of the polymer molecular weights and only considering the effect of polymer types on their solubility, the PS chains began to precipitate early and rapidly precipitated from the polymersolvent system with the solvent evaporation. The PMMA chains began to precipitate later

and slowly precipitated from the polymersolvent system because of the small differences in the solubilities of these two solvents. To reduce the surface energy, the PS chains would curl into a ball during their precipitation process as the solvent evaporated. As the surface energy of PMMA was closer to that of glass than PS and due to the interaction of the polymer-solvent system and substrate, the PS chains were precipitated into balls in the air direction. On the one hand, the PS-chain-rich phase was located away from the glass substrate. On the other hand, the PMMA-chain-rich phase was self-assembled onto the surface of the glass substrate. According to the above reasoning, the higher the ethanol content by volume, the faster the rate of precipitation of the PS chains. As the PS chains precipitated out in a short time, the process of small spherical coils binding to large particles would stop early. This phenomenon leads to a decrease in the final particle sizes with increasing ethanol content by volume. The fractal-like hierarchical micro-/nano-rough structures were formed on the copolymer surface (Figure 2f). Furthermore, the inherent hydrophobicity of PS results in a low surface energy of the copolymer. The combination of these two essential factors resulted in the copolymer surface exhibiting excellent superhydrophobicity. Figure 3 shows the cross-sectional scanning electron microscopy images of the copolymer films obtained from different ethanol contents by volume in copolymer solution: (a, b) 0\%; (c, d) 60\%. Herein, (b, d) were the higher magnification images of $(\mathrm{a}, \mathrm{c})$, respectively. The possible process of phase separation is depicted in Figure 4a. The gaps between these particles on the copolymer surfaces could effectively trap air and support the water droplets. A model of a water droplet deposited on the fractallike hierarchical micro-/ nano-rough surface of the copolymer is shown in Figure $4 \mathrm{~b}$.

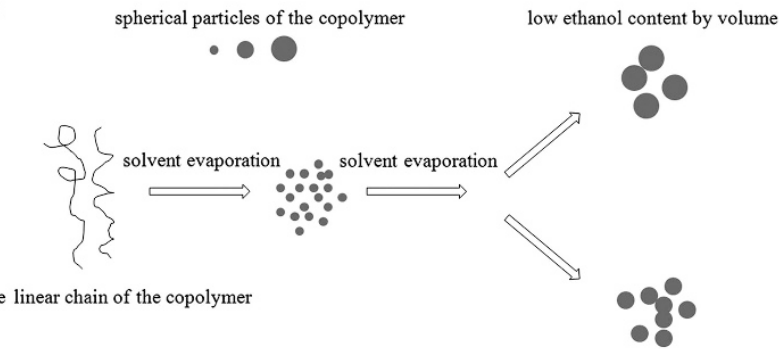

b

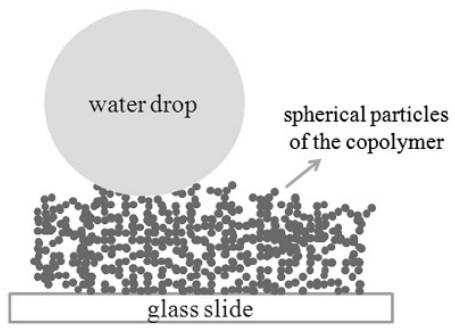
glass slide

high ethanol content by volum

Figure 4 (a) Possible phase separation process of the copolymer. (b) Model of a water drop deposited onto the fractal-like hierarchical micro-/nano-rough surface of the copolymer. A full color version of this figure is available at Polymer Journal online. 
In summary, we have described a simple method to fabricate superhydrophobic surfaces by casting PS-b-PMMA block copolymer from the mixed solvents of ethanol and THF. The diameters of the spherical particles and roughness of the copolymer surfaces could be controlled by the volume ratio of ethanol. When the ethanol content was $60 \%$ (v/v), a fractal-like hierarchical micro-/nanorough structure of the copolymer surface was obtained, and the WCA and contact angle hysteresis of this surface were $156.7^{\circ}$ and $7.1^{\circ}$, respectively. A possible phase separation process was proposed. Moreover, the films exhibited excellent self-cleaning ability that could be implemented in practice. This study enables a facile approach for preparing a superhydrophobic surface without the use of fluorine-containing substances.

\section{ACKNOWLEDGEMENTS}

This work was partially supported by the National Science and Technology Support Program of China (2009BAA23B04), National Natural Science Foundation of China (No. 60577049), Combination Project of Guangdong Province and the Ministry of Education (2011B090400397) and Luojia Young Scholars Program of Wuhan University (217273483). We thank Professor Hua Tong for providing assistance on the use of the scanning electron microscopy.

Yanfen Huang ${ }^{1}$, Xinghai Liu ${ }^{2}$, Fangfang Zhang ${ }^{2}$, Jinfeng Dong ${ }^{1}$, Yunbai Luo $^{1}$ and Chi Huang ${ }^{1,3}$
${ }^{1}$ College of Chemistry and Molecular Sciences, Wuhan University, Wuhan, PR China; ${ }^{2}$ School of Printing and Packaging, Wuhan University, Wuhan, PR China and ${ }^{3}$ Engineering Research Center of Organosilicon Compounds and Materials, Ministry of Education of China, Wuhan, PR China E-mail: chihuang@whu.edu.cn or ybai@whu.edu.cn

1 Saleema, N., Sarkar, D. K., Gallant, D., Paynter, R. W. \& Chen, X. G. Chemical nature of superhydrophobic aluminum alloy surfaces produced via a one-step process using fluoroalkyl-silane in a base medium. ACS Appl. Mater. Interfaces 3, 4775-4781 (2011).

2 Luz, G. M., Leite, A. J., Neto, A. I., Song, W. \& Mano J. F. Wettable arrays onto superhydrophobic surfaces for bioactivity testing of inorganic nanoparticles. Mater. Lett. 65, 296-299 (2010).

3 Brenier, R., Ramos, S. M. M. \& Montchanin, M. Superhydrophobic surfaces via electroless displacement of nanometric $\mathrm{Cu}$ layers by $\mathrm{Ag}+$. Appl. Surf. Sci. 255, 7439-7445 (2009).

4 Yang, J., Pi, P., Wen, X., Zheng, D., Xu, M., Cheng, J. \& Yang, Z. A novel method to fabricate superhydrophobic surfaces based on well-defined mulberry-like particles and self-assembly of polydimethylsiloxane. Appl. Surf. Sci. 255, 3507-3512 (2009).

5 Sun, T., Wang, G., Feng, L., Liu, B., Ma, Y., Jiang, L. \& Zhu, D. Reversible switching between superhydrophilicity and superhydrophobicity. Angew. Chem. Int. Ed. 43, 357-360 (2004).

6 Genua, A., Mecerreyes, D., Alduncin, J. A., Mondragon, I., Marcilla, R. \& Grande, H. -J. Polymeric ionic liquids for the fast preparation of superhydrophobic coatings by the simultaneous spraying of oppositely charged polyelectrolytes and nanoparticles. Polym. J. 43, 966-970 (2011)

7 Cabrales, L. \& Abidi, N. Microwave plasma induced grafting of oleic acid on cotton fabric surfaces. Appl. Surf. Sci. 258, 4636-4641 (2012).
8 Bhushan, B. Bioinspired structured surfaces. Langmuir 28, 1698-1714 (2012).

9 Bhushan, B. \& Her, E. K. Fabrication of superhydrophobic surfaces with high and low adhesion inspired from rose petal. Langmuir 26, 8207-8217 (2010).

10 Deng, X., Mammen, L., Butt, H. -J. \& Vollmer, D. Candle soot as a template for a transparent robust superamphiphobic coating. Science $335,67-70$ (2012).

11 Yao, X., Chen, Q, Xu, L., Li, Q, Song, Y, Gao, X Quere, D. \& Jiang, L. Bioinspired ribbed nanoneedles with robust superhydrophobicity. Adv. Funct. Mater 20, 656-662 (2010).

12 Yang, M., Di, Z. \& Lee, J. -K. Facile control of surface wettability in $\mathrm{TiO} 2 /$ poly (methyl methacrylate) composite films. J. Colloid Interface Sci. 368, 603-607 (2012).

13 Choi, B. G. \& Park, H. S. Superhydrophobic graphene/ nafion nanohybrid films with hierarchical roughness. J. Phys. Chem. C. 116, 3207-3211 (2012)

14 Wei, Z. J., Liu, W. L., Tian, D., Xiao, C. L. \& Wang, X. Q. Preparation of lotus-like superhydrophobic fluoropolymer films. Appl. Surf. Sci. 256, 3972-3976 (2010).

15 Yu, H. -J. \& Luo, Z. - H. Novel superhydrophobic silica/ poly(siloxane-fluoroacrylate) hybrid nanoparticles prepared via surface-initiated ATRP and their surface properties: the effects of polymerization conditions. $J$. Polym. Sci., Part A Polym. Chem. 49, 174-183 (2011).

16 Jafari, R., Menini, R. \& Farzaneh, M. Superhydrophobic and icephobic surfaces prepared by RF-sputtered polytetrafluoroethylene coatings. Appl. Surf. Sci. 257 1540-1543 (2010)

17 Zhang, H. \& Ruckenstein, E. Selective living anionic polymerization of a novel bifunctional monome 4-(Vinylphenyl)-1-butene and the preparation of uniform size functional polymers and amphiphilic block copolymers. Macromolecules 32, 5495-5500 (1999).

18 de Boer, B., Stalmach, U., Nijland, H. \& Hadziioannou, G. Microporous honeycomb-structured films of semiconducting block copolymers and their use as patterned templates. Adv Mater. 12, 1581-1583 (2000).

19 Tung, P. -H., Kuo, S. -W., Jeong, K. -U., Cheng, S. Z. D. Huang, C. -F. \& Chang, F. -C. Formation of honeycomb structures and superhydrophobic surfaces by casting a block copolymer from selective solvent mixtures. Macromol. Rapid Commun. 28, 271-275 (2007). 